\title{
RESEARCH
}

Open Access

\section{The effect of ursodeoxycholic acid on the relative expression of the lipid metabolism genes in mouse cholesterol gallstone models}

Ning Fan ${ }^{1 \dagger}$, Ke Meng ${ }^{2 \dagger}$, Yuqing Zhang ${ }^{3 \dagger}$, Yong Hu${ }^{4}$, Donghua $\mathrm{Li}^{5}$, Qiaoying Gao ${ }^{5}$, Jianhua Wang ${ }^{1}$, Yanning $\mathrm{Li}^{1}$, Shangwei $\mathrm{Wu}^{5}$ and Yunfeng $\mathrm{Cui}^{3^{*}}$ (D)

\begin{abstract}
Background: Many studies indicate that gallstone formation has genetic components. The abnormal expression of lipid-related genes could be the basis for particular forms of cholesterol gallstone disease. The aim of this study was to obtain insight into lipid metabolism disorder during cholesterol gallstone formation and to evaluate the effect of ursodeoxycholic acid (UDCA) on the improvement of bile lithogenicity and its potential influence on the transcription of lipid-related genes.

Methods: Gallstone-susceptible mouse models were induced by feeding with a lithogenic diet (LD) for 8 weeks. Bile and liver tissues were obtained from these mouse models after 0, 4 and 8 weeks. Bile lipids were measured enzymatically, and the cholesterol saturation index (CSI) was calculated to evaluate the bile lithogenicity by using Carey's critical tables. Real-time polymerase chain reaction (RT-PCR) was used to detect the mRNA expression levels of farnesoid $X$ receptor (FXR), liver $X$ receptor (LXR), adenosine triphosphate-binding cassette subfamily $G$ member 5/8 (ABCG5/8), cholesterol 7-a hydroxylase (CYP7A1), oxysterol 7-a hydroxylase (CYP7B1), sterol 27-a hydroxylase (CYP27A1), peroxisome proliferator-activated receptor alpha (PPAR-a) and adenosine triphosphate-binding cassette subfamily B member 11 (ABCB11).
\end{abstract}

Results: The rate of gallstone formation was 100\% in the 4-week group but only $30 \%$ in the UDCA-treated group. The UDCA-treated group had a significantly lower CSI compared with other groups. Of special note, the data on the effects of UDCA showed higher expression levels of ABCG8, ABCB11 and CYP27A1, as well as lower expression levels of LXR and PPAR-a, compared to the model control group.

\footnotetext{
* Correspondence: yfcuink@hotmail.com

${ }^{\dagger}$ Ning Fan, Ke Meng and Yuqing Zhang contributed equally to this work. ${ }^{3}$ Department of Surgery, Tianjin Nankai Hospital, Nankai Clinical School of Medicine, Tianjin Medical University, 122 Sanwei Road Nankai District, Tianjin 300100, China

Full list of author information is available at the end of the article
}

(c) The Author(s). 2020 Open Access This article is licensed under a Creative Commons Attribution 4.0 International License, which permits use, sharing, adaptation, distribution and reproduction in any medium or format, as long as you give appropriate credit to the original author(s) and the source, provide a link to the Creative Commons licence, and indicate if changes were made. The images or other third party material in this article are included in the article's Creative Commons licence, unless indicated otherwise in a credit line to the material. If material is not included in the article's Creative Commons licence and your intended use is not permitted by statutory regulation or exceeds the permitted use, you will need to obtain permission directly from the copyright holder. To view a copy of this licence, visit http://creativecommons.org/licenses/by/4.0/. The Creative Commons Public Domain Dedication waiver (http://creativecommons.org/publicdomain/zero/1.0/) applies to the data made available in this article, unless otherwise stated in a credit line to the data. 


\begin{abstract}
(Continued from previous page)
Conclusions: UDCA exhibits tremendously potent activity in restraining lipid accumulation, thus reversing the lithogenic effect and protecting hepatocytes from serious pathological damage. The abnormal expression of ABCG8, CYP7A1, CYP27A1, LXR and PPAR-a might lead to high lithogenicity of bile. These results are helpful in exploring new lipid metabolism pathways and potential targets for the treatment of cholesterol stones and for providing some basis for the study of the pathogenesis and genetic characteristics of cholelithiasis. Research on the mechanism of UDCA in improving lipid metabolism and bile lithogenicity may be helpful for clinical treatment and for reducing the incidence of gallstones.
\end{abstract}

Keywords: Cholesterol gallstone, Lipid metabolism, Ursodeoxycholic acid, ABCG8, CYP7A1, CYP27A1, LXR, PPAR-a, ABCB11

\section{Background}

It is believed that the prevalence of gallstone disease (GSD) ranges from 10 to $30 \%$, which has been observed in multiple studies $[1,2]$. Due to the improvement in living standards and irrational eating habits [3], GSD has become one of the most widespread gastroenterological conditions, imposing a considerably socioeconomic burden on health care [4]. In addition, GSD is correlated with Mirizzi syndrome and insulin resistance $[5,6]$ and can extend into the liver, gallbladder and gastrointestinal tract. More than $90 \%$ of gallstones consist mainly of cholesterol and are formed within the gallbladder [7]. It is generally thought that the key pathological abnormalities are associated with an imbalance of gallbladder bile with cholesterol supersaturation relative to bile salts. In addition, phospholipids and gallbladder hypomotility are considered as prerequisites to gallstone disease $[8,9]$.

In 1882, Dr. Langenbuch performed the first cholecystectomy, which has been considered a significant routine surgery to eradicate gallstones. With the development of surgical technology, a laparoscopic cholecystectomy has become one of the most commonly performed surgical procedures worldwide [10]. However, cholecystectomy is invasive and has a risk of postoperative complications, and not all patients with symptomatic gallstones are candidates for surgery [11]. UDCA, a natural dihydroxy bile acid, promotes gallstone dissolution and has been attributed to have several other beneficial effects, which significantly affect the treatment of cholelithiasis [12]. Currently, UDCA is recommended as a pharmacological therapy to prevent gallstone formation primarily since it is noninvasive and has fewer complications [13]. In addition, increasing studies support the notion that UDCA may enhance gallbladder muscle contractility and gastrointestinal motility to empty the gallbladder, stomach and intestine [14, 15]. In recent decades, there have been numerous investigations emphasizing the association of lipid-related genes with GSD, although little is known about how UDCA influences nuclear receptors during the process of GSD [16].

Nuclear receptors include FXR, LXR and PPAR- $\alpha$, which are hepatic lipid regulatory transcription factors of the candidate genes for gallstones $[17,18]$. It is plausible that nuclear receptors are associated with the molecular mechanism of gallstone dissolution during UDCA intervention [7]. CYP7A1 is a rate-limiting enzyme of the classic pathway of bile acid biosynthesis and plays a significant role in the alternative pathway to catalyze cholesterol into chenodeoxycholic acid (CDCA) or cholic acid (CA). FXR inhibits bile acid synthesis through downregulating CYP7A1, whereas LXR may upregulate the transcription of CYP7A1 mRNA. On the other hand, LXR regulates ABCG5 and ABCG8 to facilitate cholesterol efflux [19-21]. Moreover, adenosine triphosphate-binding cassette subfamily $\mathrm{B}$ member 4 (ABCB4) and $\mathrm{ABCB} 11$ are determinants of phospholipids (PL) and total bile acid (TBA) secretion, both of which act as flippases and efflux transporters to mediate movement of PL and TBA from hepatocytes to the canalicular membrane. TBA is reabsorbed at the terminal ileum into the portal vein to complete the enterohepatic circulation of bile acid.

This study aimed to investigate the effect of an LD and an abnormal lipid metabolism on the development of gallstones in the liver and gallbladder, histological changes in the liver, alteration in bile composition and the relative mRNA expression of genes relating to the regulation of lipid and bile acid metabolism in a mouse model of gallstone disease over a period of 8 weeks. In addition, UDCA was used in this LD-induced gallstone disease mouse model and compared with control models to evaluate a potential mechanism of action.

\section{Methods}

\section{Animals and diets}

This study was approved by the Animal Care and Use Committee in Tianjin Nankai Hospital (TMUhMEC2012019). Healthy male C57BL/6 J mice (8 weeks old, 18-20 g) were purchased from the Beijing HFK Biotechnology Company Limited (Beijing, China) and housed in plastic cages with environmentally controlled conditions $\left(22 \pm 2{ }^{\circ} \mathrm{C}\right.$, a $12 \mathrm{~h}$ light cycle) with water and food ad libitum. After adaptive feeding for 2 weeks, 60 
mice were divided randomly into 6 groups, with 10 mice in each group: LD 0-week group, LD 4-week group, LD 8-week group, LD+ normal saline (NS) 8-week group, regular rodent diet $(\mathrm{RD})+\mathrm{NS}$ 8-week group and LD + UDCA 8-week group (Table 1). The first 3 groups received an LD for 0,4 , and 8 weeks, respectively, and the last 3 groups were given the same volume of NS or UDCA $(60 \mathrm{mg} / \mathrm{kg} / \mathrm{d}$, once a day) at 9:00 am for 8 consecutive weeks. The " $0+\mathrm{LD}$ " group and "RD $+\mathrm{NS}$ " group represent the control groups. The " $8+\mathrm{LD}$ " group and "LD + NS" group represent the model groups.

The LD consisted of $80.25 \%$ regular rodent diet, $0.50 \%$ cholic acid (VETEC, Kenilworth, NJ, USA), 1.25\% cholesterol (Beijing Solarbio Science \& Technology, China), $16 \%$ butterfat and $2 \%$ corn oil. All procedures conformed to the Animal Care and Use Committee of Tianjin Nankai Hospital.

\section{Bile, gallstone, and liver sample collections}

At the end of each experiment, following an overnight fasting, the animals were anesthetized with $4 \%$ chloral hydrate. A mid-abdomen incision was made, after which liver tissues, gallstone, and bile were collected. Portions of the liver tissues were obtained for histopathological analysis and RT-PCR detection. Bile specimen was aspirated and kept for the subsequent analysis of lipid profiles. The remaining liver tissues and gallstones were stored at $-80^{\circ} \mathrm{C}$ (Thermo Scientific Forma, Waltham, MA, USA) for further assessment. After the collection of samples, the mice were euthanized.

\section{Hematoxylin and eosin (HE) staining}

Liver tissues were fixed with $4 \%$ neutral buffered formaldehyde for 2 weeks. The fixed tissues were dehydrated, embedded in paraffin, and serially sectioned into $4 \mu \mathrm{m}$ slices to eventually achieve HE staining for subsequent observation under an upright microscope (Nikon, Chiyoda ward, Kyoto, Japan).

\section{Analysis of bile lipids}

Bile total cholesterol (TC), PL and TBA (the TC diagnostic kit was purchased from Roche Diagnostics Ltd., Basel, Switzerland; the PL kit was purchased from Beijing Leadman Biochemical Co, Ltd., China; the TBA

Table 1 Mouse grouping and feeding schedule

\begin{tabular}{lll}
\hline Groups & Number & Feeding time \\
\hline $0+$ LD & 10 & 0 week \\
$4+$ LD & 10 & 4 weeks \\
$8+$ LD & 10 & 8 weeks \\
RD + NS & 10 & 8 weeks \\
LD + NS & 10 & 8 weeks \\
LD + UDCA & 10 & 8 weeks \\
\hline
\end{tabular}

kit was purchased from Beijing Strong Biotechnologies, Inc., China) were detected by an automatic biochemical analyzer (Cobas 8000, Basel, Switzerland). The CSI was calculated according to Carey's critical tables [22].

\section{RT-PCR detection}

Total RNA was extracted by using an RNAprep Pure Tissue Kit (Tiangen, Beijing, China). To harvest cDNA, $2 \mu \mathrm{g}$ RNA and the Oligo (dT) 18 primer was used in the reverse transcription reaction using the RevertAid ${ }^{\mathrm{Tm}}$ First Strand cDNA Synthesis Kit (Thermo, USA). Amplification was performed on a RT-PCR detection system (BioRad IQ5, Waltham, MA, USA) with the GoTaq ${ }^{\circ}$ qPCR Master Mix (Promega, Madison, Wisconsin, USA). The relative expression levels of genes were quantified by using the $2^{-\Delta \Delta \mathrm{Ct}}$ method, and glyceraldehyde-3phosphate dehydrogenase (GAPDH) was deemed as the invariant control. Ct represents cycle threshold. The forward and reverse primers of the mouse genes were designed for RT-PCR and gel electrophoresis as follows:

FXR (5' 'TGGGTACCAGGGAGAGACTG-3' and 5' GTGAGCGCGTTGTAGTGGTA-3'),

LXR (5'-AGACGTCACGGAGGTACAAC-3' and 5'AGCAGAGCAAACTCAGCATC-3'),

ABCG5 (5'-GGAGAACATTGAAAGAGCAC-3' and 5'-GTTACTCGCCTCAGCAG-3'),

ABCG8 (5'-GACAGCTTCACAGCCCACAA-3' and 5'-GCCTGAAGATGTCAGAGCGA-3'),

CYP7A1 (5'-CTTCATCACAAACTCCCTGTC-3' and 5'-GTCCAAATGCCTTCGCAG-3'),

CYP7B1 (5' -CCGATTCTGCCGTCTCCTT-3' and 5' CCAGCCTTACTCTGCAAAGCTT-3'),

CYP27A1 (5'-GATCTTCATCGCACAAGGAG-3' and 5'-GATAACCTCGTTTAAGGCATCC-3'),

PPAR- $\alpha$ (5'-TGGTTGAATCGTGAGGAACA-3' and 5'-ATCGCCACTAAGGTGTCAGG-3'),

ABCB11 (5'-AATAGACAGGCAACCCGT-3' and 5' GAGAAGGATAATGGAAGGTCAC-3').

In addition, the relative expression levels of the genes' mRNAs were normalized to GAPDH mRNA.

\section{Statistical analysis}

The data were presented as the mean \pm standard deviation. A chi-square test was used to compare the stone formation rate among the control group, model group and LD + UDCA group. Comparisons among the different groups were determined by one-way analysis of variance with least significant difference (LSD) and StudentNewman-Keuls (SNK) analysis. The enumeration data were expressed as a percentage. Statistical analysis was delineated on SPSS 17.0 (SPSS, Chicago, IL, USA) and GraphPad Prism 6.0 (GraphPad Software, La Jolla, CA, USA). A difference was considered statistically significant when the two-sided tests showed a $P$ value of less 
than 0.05 and extremely significant when $P<0.01$ or $P<$ 0.001 . A Spearman correlation coefficient analysis was performed to correlate bile lipid concentrations with gene expression levels.

\section{Results}

\section{The evolution of gallstone formation}

The conditions of the liver and gallbladder with or without gallstones were recorded completely in macroscopic and microscopic evaluations $(2 \times$ stereo, Olympus, Kyoto, Japan) and are pictured in Fig. 1.

The conditions in the samples of the $0+\mathrm{LD}$ and $\mathrm{RD}+$ NS groups appeared to be normal, which showed dark red liver tissues and transparent gallbladders with no flowing gallstones, floccules or crystals. In the $4+\mathrm{LD}$ group, mild changes appeared in the liver, with a shallow color, crisp texture and serrated edge, while the gallbladders were significantly altered with cholecystectasia and cholestasis, accompanied by flocculent sediment. In the model groups, massive granulated white and light-yellow gallstones floated in bile, and dramatic changes occurred in the liver, which turned gray or white with a greasy appearance. Otherwise, UDCA reversed the evolution of gallstone formation, improving the conditions of cholecystectasia and cholestasis, which were similar to the $4+$ LD group.

\section{Gallstone prevalence}

The preliminary experiment was performed on 10 C57BL/6 J mice on an LD for 8 weeks to obtain a successful mouse model of cholesterol gallstones. As shown in Fig. 2, the gallstone prevalence in the $4+$ LD group, control group and model group were 50, 0 and 100\% (5/ $10,0 / 10$, and 10/10), respectively. The prevalence rate of the LD + UDCA group was 30\% (3/10), which was significantly lower than that of the LD + NS group.
Floccules were viewed as the transition state of gallstone formation, and the prevalence in both the $4+\mathrm{LD}$ group and LD + UDCA group was 10\% (1/10). Statistical analysis showed that the prevalence of gallstones was significantly different among the model group, control group and LD + UDCA group $(P<0.05)$.

\section{Qualitative analysis of gallstones}

Gallstones were collected and analyzed by Fourier transform infrared spectroscopy (Thermo Scientific Forma, Waltham, MA, USA). The collected gallstones were washed and dried to perform qualitative analysis with Fourier transform infrared spectroscopy, and a strong absorption peak of the cholesterol gallstone was detected. There were strong absorption peaks detected at 2930, 1466, 1382 and $1055 \mathrm{~cm}^{-1}$, which aligned with the cholesterol absorption peaks. There was no characterized pigment absorption peak, indicating that the gallstones can be classified as cholesterol gallstones.

\section{Morphological observation}

As a result of the LD, the histopathology of the liver tissue was worse than that observed in the control groups (Fig. 3). Fatty degeneration of the liver cells, ballooning degeneration of the liver cells or hepatocellular hyperplasia could be markedly observed in the model groups. Regarding reduction in the fatty droplets in the hepatocytes, the morphological change in the LD + UDCA group and the $4+\mathrm{LD}$ group fell between that of the control group and the model group.

\section{Bile lipid levels}

The concentrations of the bile lipids are summarized in Fig. 4. The data indicated that the mice that were fed an LD may have a great possibility of increasing bile indicators, such as TC, PL and CSI, all of which had

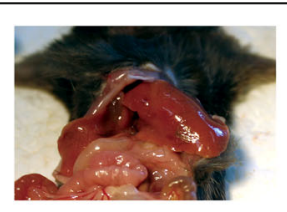

a

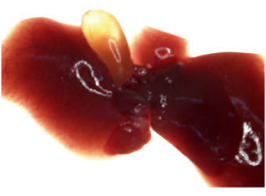

e

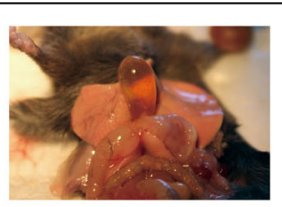

b

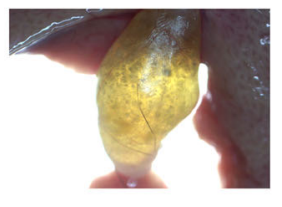

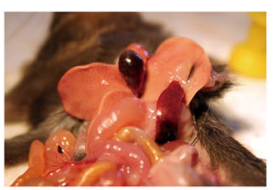

c

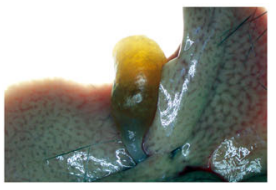

g

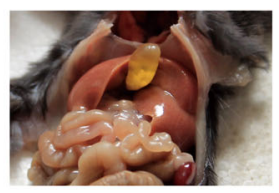

d

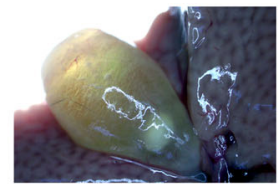

Fig. 1 The evolution of gallstone formation in the liver and gallbladder. $\mathbf{a}, \mathbf{b}, \mathbf{c}$, and $\mathbf{d}$ : the macroscopic anatomic structure of the liver and gallbladder; e, f, $\mathbf{g}$, and $\mathbf{h}$ : images from the $2 \times$ stereo microscope; $\mathbf{a}$ and $\mathbf{e}$ : normal liver and gallbladder; b: cholecystectasia; $\mathbf{f}$ : floccules; $\mathbf{c}$ and $\mathbf{g}$ : cholestasis; $\mathbf{d}$ and $\mathbf{h}$ : massive gallstone 


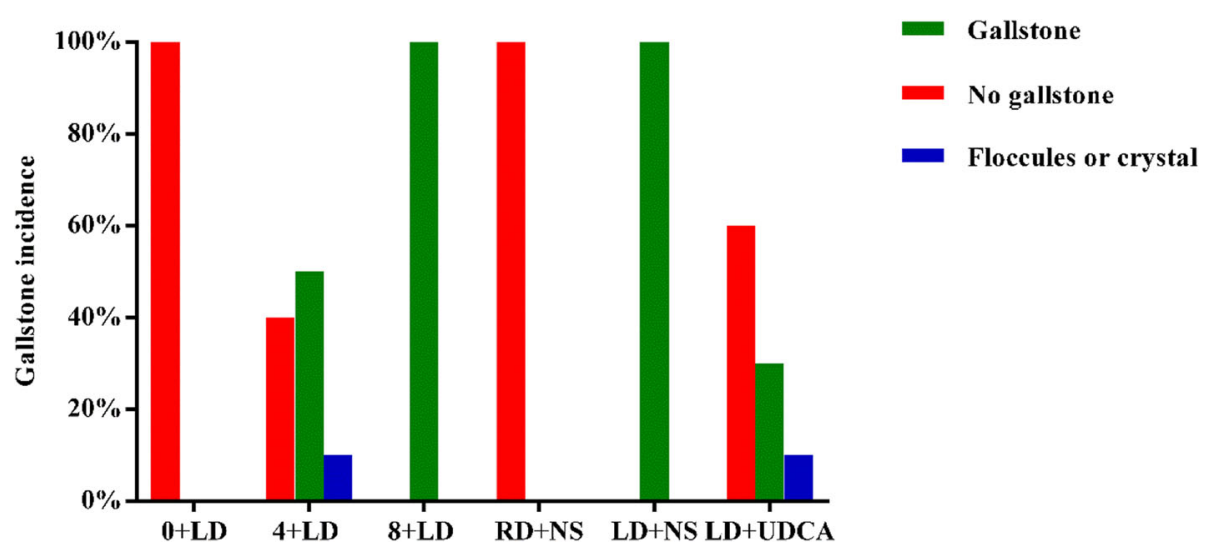

Fig. 2 Gallstone prevalence in each group $(n=10)$

significantly higher concentrations in the $8+\mathrm{LD}$ group and the $4+\mathrm{LD}$ group in comparison with the $0+\mathrm{LD}$ group. In the LD + UDCA group, the levels of TC, PL and CSI in the bile were significantly reduced when compared with the model group. All these results demonstrated that UDCA may play a key role in improving the CSI and bile lipids.

\section{The expression of lipid-related genes in the liver}

Mice in the $4+\mathrm{LD}$ and $8+\mathrm{LD}$ groups showed higher expression levels of ABCG5 $(P=0.002)$ and PPAR- $\alpha(P<$ $0.001)$ with respect to the $0+\mathrm{LD}$ group, as shown in the results in Fig. 5. The expression levels of CYP7A1 $(P<$ $0.001)$ and CYP7B1 $(P<0.001)$ showed significant reductions in the $4+\mathrm{LD}$ group and elevations in the $8+\mathrm{LD}$ group, conversely. ABCG8 $(P<0.001)$ was upregulated in the $4+\mathrm{LD}$ group and downregulated in the $8+\mathrm{LD}$ group. The expression levels of ABCB11 mRNA among the $0+\mathrm{LD}, 4+\mathrm{LD}$, and $8+\mathrm{LD}$ groups were significantly different $(P=0.02)$.

The results showed that there was a higher level of ABCG8 $(P<0.001)$ and CYP27A1 $(P=0.033)$, as well as a lower level of LXR $(P=0.026)$ and PPAR- $\alpha(P=0.015)$ in the LD + UDCA group compared to the LD + NS group. The expression of ABCB11 mRNA among the $\mathrm{LD}+\mathrm{NS}, \mathrm{RD}+\mathrm{NS}$ and $\mathrm{LD}+\mathrm{UDCA}$ groups were also statistically significant $(P=0.008)$. However, there was no significant difference in the expression of ABCG5, CYP7A1, CYP7B1, and FXR between the LD + NS group and the LD + UDCA group (Fig. 6).

The hierarchical cluster analysis and heatmap representation of mRNA expression levels in ABCG5/8, ABCB11, CYP7A1, CYP7B1, CYP27A1, FXR, LXR, and PPAR- $\alpha$ (Fig. 7).

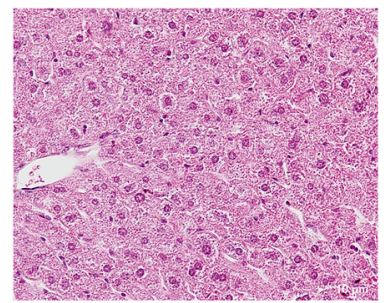

A

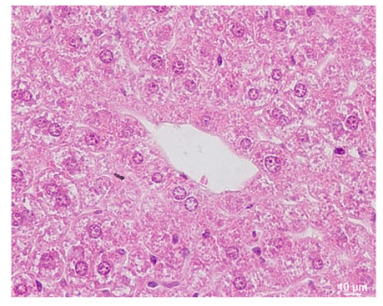

a

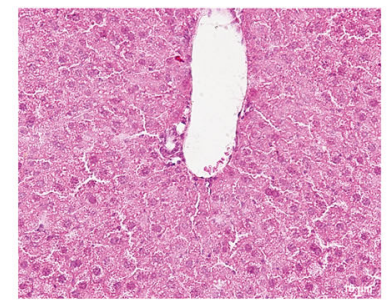

B

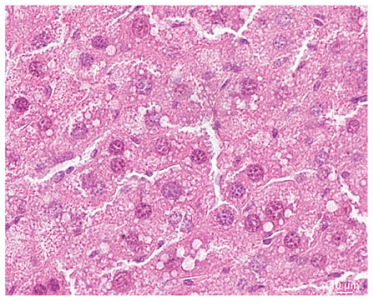

b

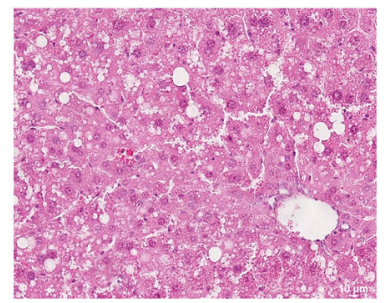

C

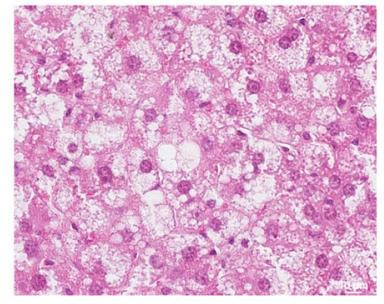

Fig. 3 HE staining of liver tissues. $\mathbf{A}$ and $\mathbf{a}$ : normal liver cell; $\mathbf{B}$ and $\mathbf{b}$ : cell in transition stage; $\mathbf{C}$ and $\mathbf{c}$ : cell with serious damage. The magnifications are 200x in A, B and $\mathbf{C}$ and 400x in $\mathbf{a}, \mathbf{b}$ and $\mathbf{C}$ 


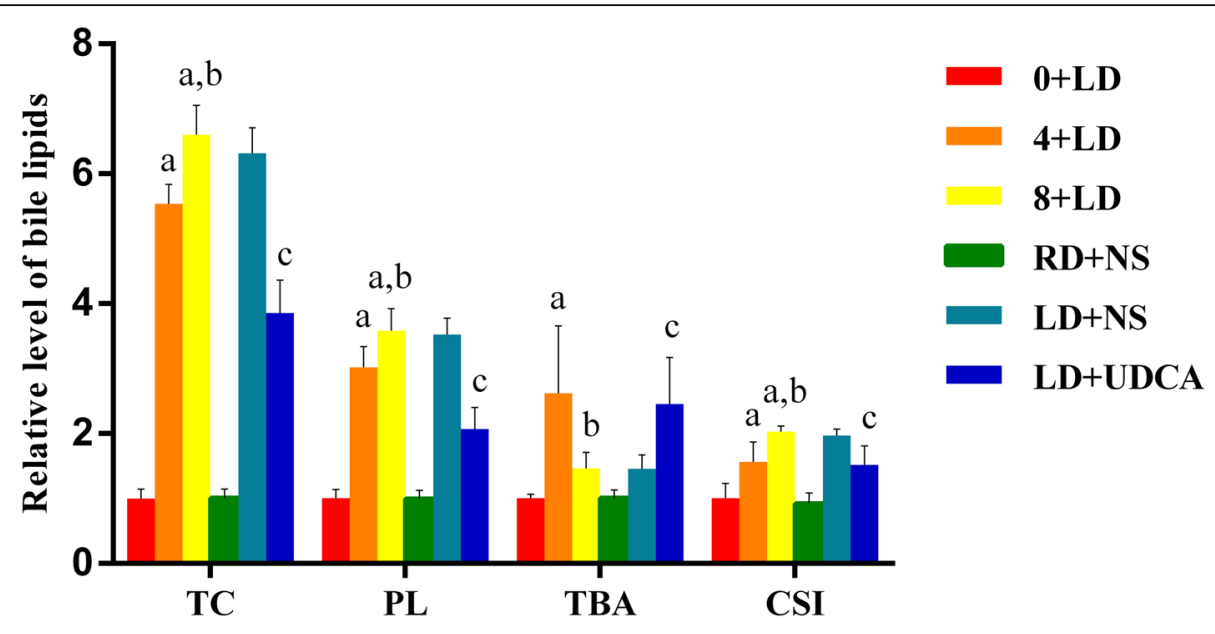

Fig. 4 Relative levels of the bile lipids. a, significantly different from the control group $(P<0.001)$; $b$, significantly different from the $4+L D$ group $(P<0.001)$; $C$, the $P$ value in LD + UDCA group was significantly different from that of the control group $(P<0.001)$. The data of the lipid contents of the control group were set to 1

The UDCA-dependent gene expression relationship is shown in Fig. 8.

\section{The correlation analysis between gene expression and bile lipids}

A Spearman correlation coefficient analysis was performed to correlate bile lipid concentrations with gene expression levels. The results showed that ABCG5 and ABCG8 positively correlated with TC, PL and TBA in bile, CYP7A1 and CYP7B1 negatively correlated with TC, PL and TBA, and PPAR- $\alpha$ positively correlated with $\mathrm{TC}$ and PL, all of which were statistically significant differences (Table 2).

\section{Discussion}

In this study, gallstone-susceptible C57BL/J mice successfully expressed a $100 \%$ gallstone prevalence after feeding on an LD for 8 weeks. The results of this study are consistent with those of Liu [23]. Due to its abundance of fat and cholesterol, a lithogenic diet induced cholesterol gallstones in the mice by progressively influencing the imbalance of lipid metabolism with higher TC and PL, thus giving rise to a higher bile CSI and cholesterol crystallization until gallstone formation and acceleration of the deterioration of the liver into fatty liver occurred. The gallstone prevalence and liver deterioration were gradually increased with the extension of feeding time. Of note, mice that had been administered UDCA showed a lower gallstone prevalence of $30 \%$. These results reinforce the findings from previous studies that UDCA may exhibit a tremendously potent activity in increasing the bile acid pool size and thus reduce the cholesterol saturation index and cholesterol gallstone formation.

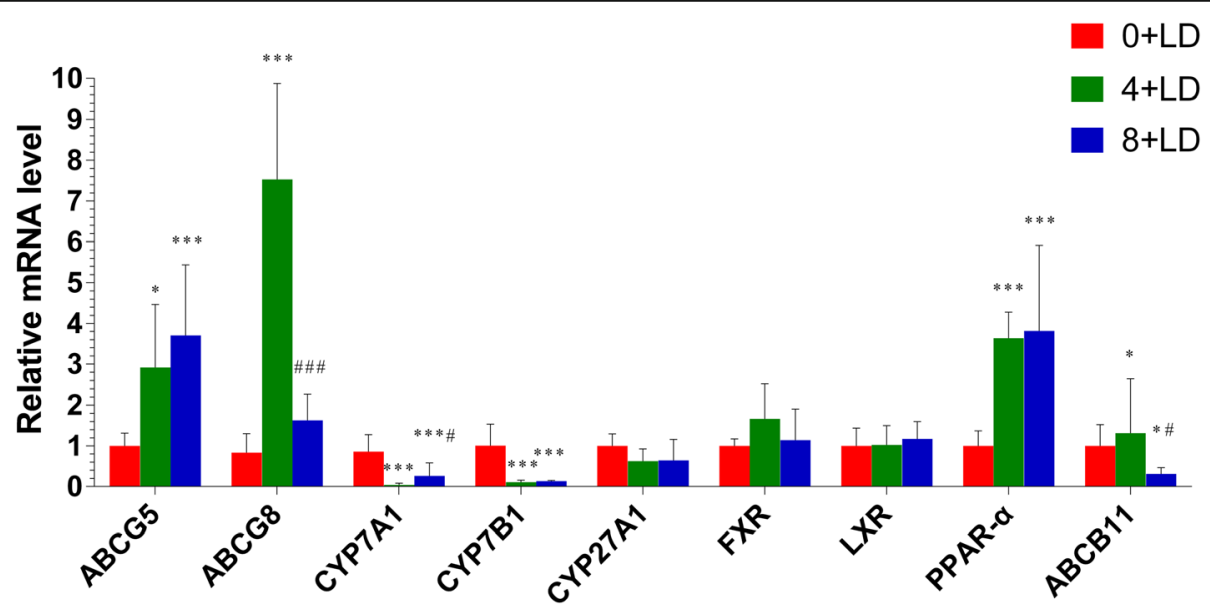

Fig. 5 Relative mRNA expression levels of lipid-related genes in the $0+L D$ group, $4+L D$ group and $8+L D$ group assessed using RT-PCR. * compared to the $0+$ LD group; \#, compared to the $4+$ LD group; ${ }^{*}, \#, P<0.05 ;{ }^{* *}, \# \#, P<0.01$; ***, \#\#\#, $P<0.001$ 

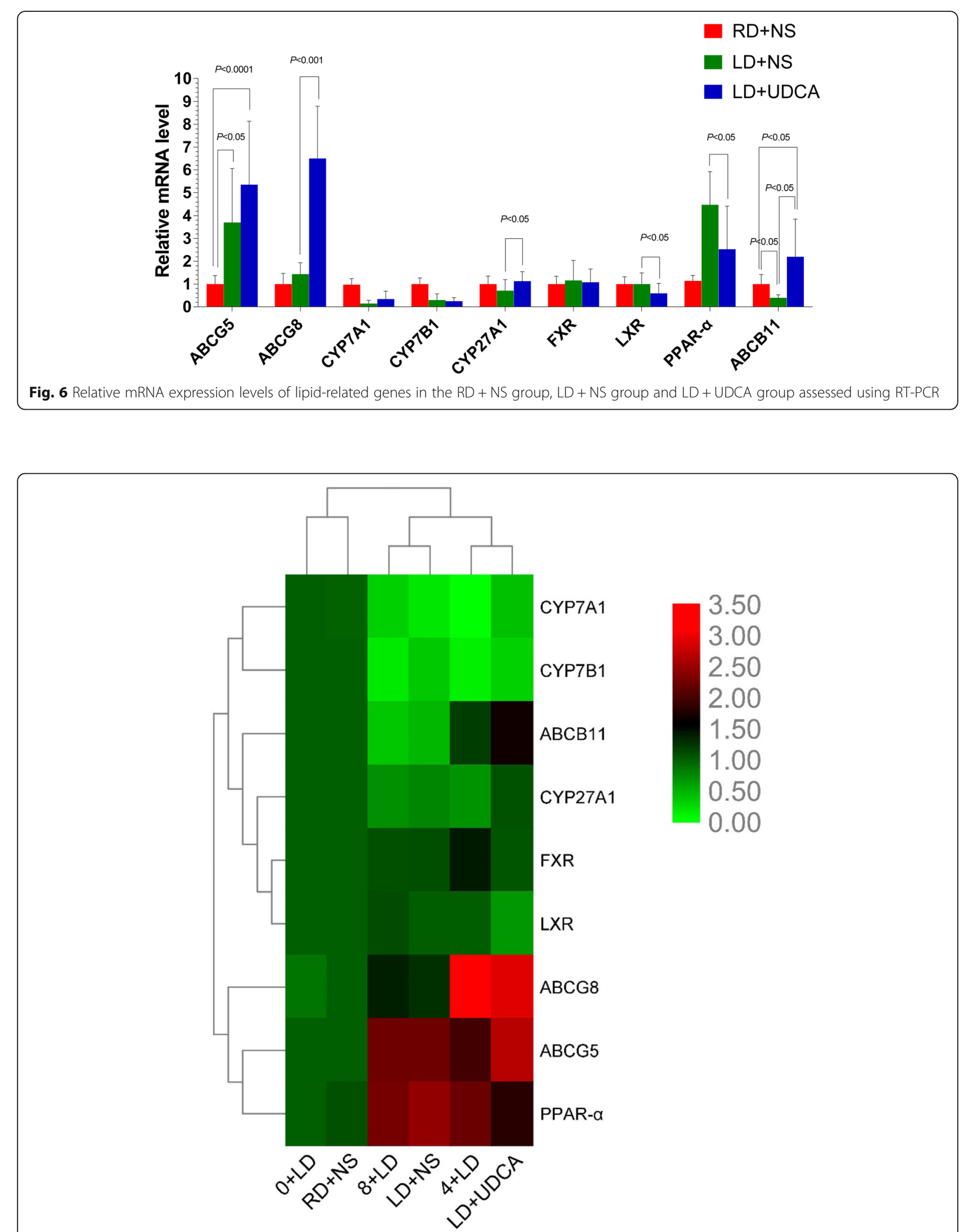

Fig. 7 Heatmap representation of mRNA expression levels 


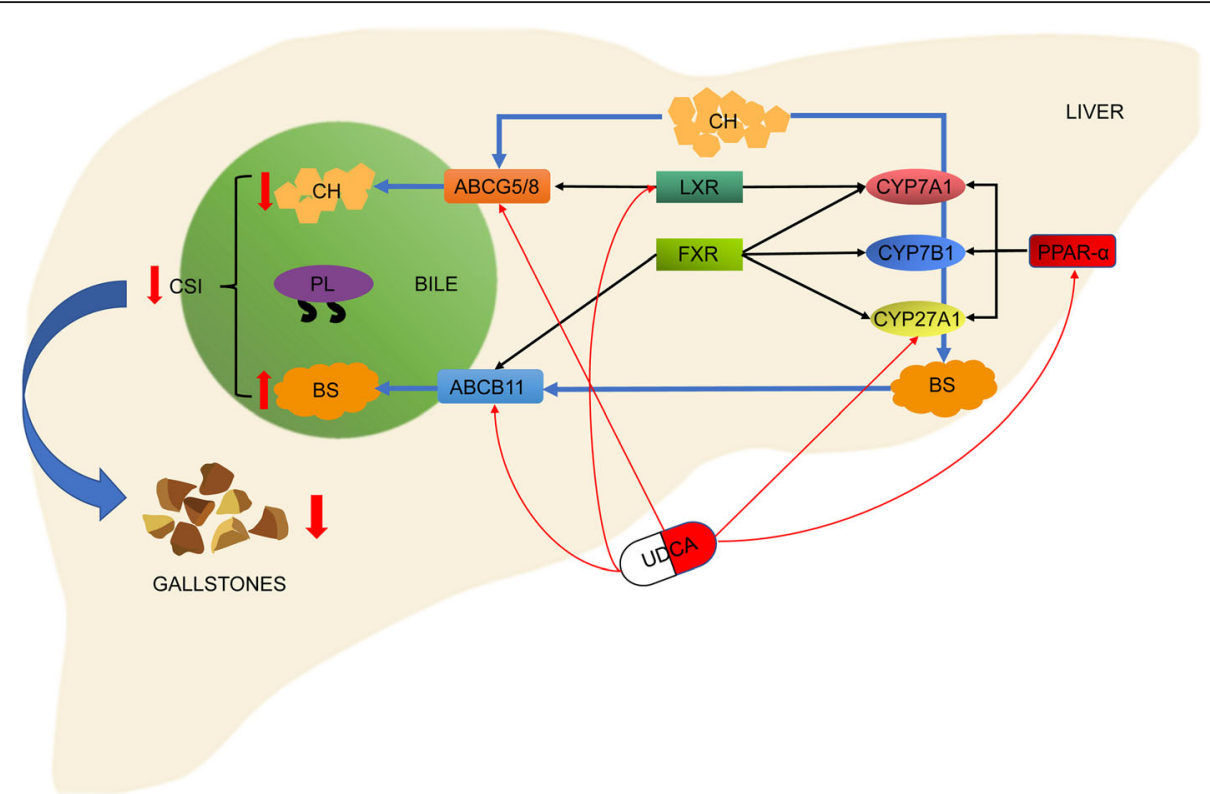

Fig. 8 The figure shows the schematic diagram of cholesterol $(\mathrm{CH})$ and bile salt (BS) metabolism regulated by the genes involved in this study, as well as the genes that UDCA might affect

ABCG5/8 gene products, predominantly localized on the canalicular membrane of hepatocytes, are heterodimerized to function as a sterol transporter to facilitate cholesterol secretion into the bile canaliculus. The ABCG5/8 genes are transcriptionally regulated under the activation of LXR, and the overexpression of the ABCG5/8 genes is attributed to GSD [24-26]. Biliary cholesterol secretion is primarily maintained by $\mathrm{ABCG} 5 / 8$, while recent investigation insists that almost $30 \%$ of the cholesterol output is transferred via an ABCG5/8-independent pathway [27]. Lithogenic food could induce the excess secretion of cholesterol and oxysterol congeners, thus adversely affecting the expression of ABCG5/8.

The expression of ABCG5 mRNA in the $4+\mathrm{LD}$ and $8+$ LD groups showed a significantly higher tendency with respect to the $0+\mathrm{LD}$ group. Although the expression of LXR increased gradually with the extension of LD feeding time, the difference was not significant. For ABCG8, there was a significantly increased level in the $4+$ LD group and a decrease in the $8+$ LD group. Conversely, UDCA may have a potential ability to enhance the transcription of ABCG8 and to decrease LXR, compared to the model group. Bile acids are thought to regulate their own homeostasis via their sensor, the FXR, which could be directly activated by UDCA [28]. LXR are "cholesterol sensors" which, in response to excess cholesterol, stimulate its transport to the liver and biliary excretion [17]. The possible mechanism for this is that when UDCA enters the bile, it changes the bile salt concentration and increases cholesterol solubility. On one hand, UDCA can dissolve cholesterol stones/crystals in bile, and on the other hand, it can also stimulate ABCG8 to transport cholesterol forward into the bile. Therefore, the expression of ABCG8 is increased. LXR, as a cholesterol receptor, was passively downregulated to maintain cholesterol homeostasis when cholesterol transport increased. In addition, studies have shown that the ABCG5/8-independent pathway plays a critical role in regulating hepatic cholesterol secretion and in determining the susceptibility to cholesterol gallstones, working independently of the ABCG5/8 pathway, and that the hepatic LXR does not have an effect on the ABCG5/ 8-independent pathway for regulating biliary cholesterol secretion [29]. This is different from the classical mechanism of the LXR-ABCG5/8 pathway in regulating cholesterol metabolism. Because the concentration of bile

Table 2 Correlation analysis between gene expression and bile lipids

\begin{tabular}{|c|c|c|c|c|c|c|c|c|c|c|c|c|c|c|c|c|}
\hline \multirow[t]{2}{*}{ Bile lipid } & \multicolumn{2}{|c|}{ ABCG5 } & \multicolumn{2}{|c|}{ ABCG8 } & \multicolumn{2}{|l|}{ CYP7A1 } & \multicolumn{2}{|l|}{ CYP7B1 } & \multicolumn{2}{|c|}{ CYP27A1 } & \multicolumn{2}{|l|}{ FXR } & \multicolumn{2}{|l|}{$\underline{L X R}$} & \multicolumn{2}{|c|}{ PPAR-a } \\
\hline & $r$ & $P$ & $r$ & $P$ & $r$ & $P$ & $r$ & $P$ & $r$ & $P$ & $r$ & $P$ & $r$ & $P$ & $r$ & $P$ \\
\hline TC & 0.783 & 0.000 & 0.477 & 0.014 & -0.618 & 0.001 & -0.633 & 0.001 & -0.134 & 0.515 & -0.060 & 0.772 & 0.132 & 0.520 & 0.696 & 0.000 \\
\hline$P L$ & 0.617 & 0.001 & 0.467 & 0.016 & -0.628 & 0.001 & -0.618 & 0.001 & -0.259 & 0.202 & 0.031 & 0.886 & 0.204 & 0.318 & 0.726 & 0.000 \\
\hline TBA & 0.613 & 0.001 & 0.674 & 0.000 & -0.534 & 0.005 & -0.453 & 0.020 & 0.077 & 0.710 & -0.142 & 0.490 & -0.107 & 0.603 & 0.253 & 0.212 \\
\hline
\end{tabular}


salt is limited, when the concentration of bile salt reaches a constant level, it can affect the solubility of cholesterol, leading to cholesterol crystallization/stone formation. It may also be that UDCA is not effective for every patient or that stones recur after a period of time. Of course, many studies are needed to verify this in a follow-up study.

It is known that there are two pathways for the elimination of redundant cholesterol, where one is via the LXR-ABCG5/8 pathway to bile and the other is via bile acid synthesis to convert cholesterol into bile acid. One possible reason for the results obtained is that in response to persistent hypersecretion of cholesterol or oxycholesterol, the feed-forward loop may become active and the LXR mRNA may be motivated to decrease the cholesterol level in hepatocytes [30]. An increase in cholesterol can lead to an increase in oxysterols, which activates LXR to induce CYP7A1 and ABCG5/8 expression. The activation of LXR significantly influences ABCG5/8, thus increasing the transcription of ABCG5/ 8 . The possible reason is that ABCG8 may contribute to the peak of the lithogenic effect after 4 weeks of lithogenic food. When the mice were fed with LD for 4 weeks, the expression of ABCG8 increased significantly, which was associated with the activation of this classical pathway. However, it is important to mention that LXR only induces CYP7A1 in rats and mice and not in hamsters, rabbits or humans [31].

It is known that excess cholesterol may participate in bile acid synthesis, which is coordinated by CYP7A1 and CYP27A1 [32]. These are two critical enzymes in the classic and alternative pathways to regulate cholesterol trafficking into CDCA or CA, which are concomitant with their downstream target genes to sustain cholesterol homeostasis. ABCB11 is a bile salt export pump that facilitates the efflux of bile acid to the canalicular membrane, thereby performing enterohepatic circulation of bile acid. Additionally, it was widely believed that the upregulation of LXR may exert a beneficial effect on the elevation of CYP7A1 expression, while FXR as a bile acid receptor may suppress CYP7A1 [33, 34]; however, in this experiment, no significant difference was observed in the FXR mRNA. It is thus controversial whether or not PPAR- $\alpha$ may downregulate CYP7A1 and CYP27A1 mRNA in fatty acid catabolism [35-37]. In the present study, the results suggested that the upregulation of PPAR- $\alpha$ may be implicated in the downregulation of CYP7A1. This is consistent with Xie's research results [38]. In the LD-fed L-Fabp-/- mice, in the absence of LFabp (whose expression is generally regulated in a proximal to distal gradient), alterations in intestinal BA flux induce FXR expression, which results in transcriptional induction of downstream targets, including fibroblast growth factor (FGF) 15 [39]. Increased intestinal FGF15 expression would in turn be anticipated to suppress hepatic CYP7A1 expression [40, 41]. Thereafter, there was a remarkable finding that CYP7A1 and CYP7B1 mRNA showed downregulation at 4 weeks and, conversely, upregulation at 8 weeks, while PPAR- $\alpha$ may show a sustainable increase from 0 to 8 weeks. A different discrepancy in CYP7A1 may be attributed to the common control of LXR, FXR and PPAR- $\alpha$, while CYP27A1 may be affected by the higher expression of PPAR- $\alpha$. A prolonged lithogenic diet may produce serious injury to hepatocytes or the canalicular membrane, thus leading to the decrease in the CYP7A1, CYP7B1 or CYP27A1 translocators. Recently, studies have shown that UDCA may be an FXR antagonist [42, 43]. In liver, the majority of UDCA is conjugated with glycine and taurine to produce glycoursodeoxycholic acid (GUDCA) and tauroursodeoxycholic acid (TUDCA), which are transported into the gut and then reabsorbed into the ileum epithelial cells. This study revealed that GUDCA and TUDCA were bona fide FXR antagonists. Oral administration of GUDCA suppressed FXR signaling in the gut but did not affect FXR signaling in the liver. Because UDCA was found not to be a direct FXR antagonist, the metabolic benefits of UDCA may be due in part to GUDCA [44].

ABCB11 mRNA expression showed a trend of an increase first, followed by a decrease, among the $0+\mathrm{LD}$, $4+\mathrm{LD}$, and $8+\mathrm{LD}$ groups. This may be related to the influence of other factors on the expression of ABCB11, such as steroid receptor coactivator-2 (SRC-2), liver kinase B1 (LKB1), liver receptor homolog 1 (Lrh1), NF-E2related factor-2 (Nrf2), and others [45-47]. Moreover, the expression of ABCB11 mRNA in the LD + UDCA group was significantly higher than that in the RD + NS and LD + NS groups. This indicated that under UDCA intervention, the expression of ABCB11 mRNA was upregulated, which was consistent with the results of $\mathrm{Hu}$ et al. The ABCB11 gene encodes a protein called the bile salt export pump, which transports bile salts from the hepatocyte into bile. The overexpression of ABCB11 significantly promoted biliary bile salt secretion and increased the circulating bile salt pool size and bile saltdependent bile flow rate [48]. After that, cholesterol solubility can be increased, thus lowering the CSI and reducing cholesterol stone formation. The increased expression of ABCB11 could increase the rate of transport of bile salts within the hepatocyte, thereby decreasing their steady-state intracellular concentration and thus leading to enhanced signaling [49]. As a natural ligand of liver FXR, bile acid can play a role as a signaling molecule regulating liver metabolism [50]. Further studies are needed to confirm the possibility that lipid changes in bile may affect the expression of some genes.

To date, it has been discovered that the CYP27A1, LXR, PPAR- $\alpha, A B C G 8$ and ABCB11 genes involved in 
lipid metabolism predispose an individual to gallstone formation [51, 52]. However, their interactions with UDCA have not been fully ascertained yet.

\section{Strength and study limitation}

This study only examined gene expression in liver tissue and did not detect the same genes or undetected target genes in sites such as the gallbladder and intestines. This study was restricted to in vitro experiments, and the protein levels of the lipid-related genes should be further determined to verify the conclusions.

In the future, more basic experiments are needed to study the specific physiological mechanism of UDCA treatment of cholesterol stones. The protein levels of these genes need to be tested, and it needs to be confirmed whether these results in vitro have potential value for research on the mechanism, prevention and treatment of human cholelithiasis. Future research should ultimately be concentrated on the definitive molecular and pharmacokinetic mechanism by which UDCA may affect certain lipid-related genes to assess the therapeutic feasibility of gene agonists that may be applied to GSD.

\section{Conclusions}

With regard to the mouse groups on an LD for 0,4 , and 8 weeks, it should be emphasized that these alterations in the lipid-related genes may accelerate cholesterol accumulation and bile supersaturation while modulating bile lipids and the CSI to exert significant effects on gallstone formation. UDCA may achieve a cholagogic effect by decreasing the levels of TC, PL and CSI to normalize lipid composition. Thus, increasing the expression levels of ABCG8, ABCB11, CYP7A1 and CYP27A1 or decreasing the levels of LXR and PPAR- $\alpha$ can stimulate bile acid secretion and efficiently facilitate gallstone dissolution to reverse damage to the hepatocytes. UDCA may play a role in regulating the expression levels of some lipidrelated genes directly or indirectly to ameliorate the development of gallstones. Therefore, UDCA can be used as the drug of choice for cholesterol gallstones if patients are unwilling to undergo surgery.

\footnotetext{
Abbreviations

UDCA: Ursodeoxycholic acid; LD: Lithogenic diet; CSI: Cholesterol saturation index; RT-PCR: Real-time polymerase chain reaction; GSD: Gallstone disease; FXR: Farnesoid X receptor; LXR: Liver X receptor; PPAR-a: Peroxisome proliferator activated receptor alpha; CYP7A1: Cholesterol 7-a hydroxylase; CYP27A1: Sterol 27-a hydroxylase; ABCB11: Adenosine triphosphate-binding cassette subfamily B member 11; CDCA: Chenodeoxycholic acid; CA: Cholic acid; ABCG5: Adenosine triphosphate-binding cassette subfamily $G$ member 5; ABCG8: Adenosine triphosphate-binding cassette subfamily $G$ member 8; ABCB4: Adenosine triphosphate-binding cassette subfamily $B$ member 4; PL: Phospholipids; TBA: Total bile acid; NS: Normal saline; RD: Regular rodent diet; HE: Hematoxylin eosin; TC: Total cholesterol; GAPDH: Glyceraldehydes-3phosphate dehydrogenase; LSD: Least significant difference; SNK: Studentnewman-keuls; CYP7B1: Oxysterol 7-a hydroxylase; FGF15: Fibroblast growth factor 15; GUDCA: Glycoursodeoxycholic acid; TUDCA: Tauroursodeoxycholic
}

acid; SRC-2: Steroid receptor coactivator-2; LKB1: Liver kinase B1); Lrh1: LIVER receptor homolog 1; Nrf2: NF-E2-related factor-2; $\mathrm{CH}$ : Cholesterol; BS: Bile salt

\section{Acknowledgements}

Not applicable.

\section{Authors' contributions}

YFC and SWW conceived and designed the study. KM, DHL and QYG performed the experiments. NF, KM and YQZ wrote the paper. NF and $\mathrm{YH}$ reviewed and edited the manuscript. The author(s) read and approved the final manuscript.

\section{Funding}

This study was funded by Natural Science Foundation of Tianjin Bureau of traditional Chinese Medicine13040, Key project of Tianjin science and technology support plan14ZCZDSY00021, Foundation of Tianjin Clinical Medical Research Center of Acute Abdomen with integrated Chinese and Western medicine15ZXLCSY00030, Project of the national science and technology program of Ministry of human resources and social security, Project of Tianjin thousands of people plan.

These funding sources are non-commercial.

\section{Availability of data and materials}

The datasets used and/or analysed during the current study are available from the corresponding author on reasonable request.

\section{Ethics approval and consent to participate}

All procedures conformed to the National Institutes of Health guidelines and were approved by the Animal Care and Use Committee in Tianjin Nankai Hospital.

\section{Consent for publication}

Not applicable.ik

\section{Competing interests}

The authors declare that they have no competing interests.

\section{Author details}

${ }^{1}$ Beichen Chinese Medicine Hospital Affiliated to Tianjin University of Traditional Chinese Medicine, 436 Jingjin Road, Beichen District, Tianjin 300400, China. 'Department of Obstetrics and Gynecology, General Hospital of Tianjin Medical University, 154 AnShan Road, HePing District, Tianjin 300052, China. ${ }^{3}$ Department of Surgery, Tianjin Nankai Hospital, Nankai Clinical School of Medicine, Tianjin Medical University, 122 Sanwei Road Nankai District, Tianjin 300100, China. ${ }^{4}$ Tianjin Medical University, 22 Qixiangtai Road, Heping District, Tianjin 300070, China. ${ }^{5}$ Institute of Acute Abdomen in Integrative Medicine, Tianjin Nankai Hospital, Nankai Clinical School of Medicine, Tianjin Medical University, 122 Sanwei Road Nankai District, Tianjin 300100, China.

Received: 12 February 2020 Accepted: 23 June 2020 Published online: 02 July 2020

\section{References}

1. Chen YC, Chiou C, Lin MN, Lin CL. The prevalence and risk factors for gallstone disease in taiwanese vegetarians. PLoS One. 2014;9:e115145.

2. Miquel JF, Covarrubias C, Villaroel L, Mingrone G, Greco AV, Puglielli L, et al. Genetic epidemiology of cholesterol cholelithiasis among chilean hispanics, amerindians, and maoris. Gastroenterology. 1998;115:937-46.

3. Gong Y, Li S, Tang Y, Mai C, Ba M, Jiang P, et al. Cholelithiasis and risk of pancreatic cancer: systematic review and meta-analysis of 21 observational studies. Cancer Causes Control. 2014;25:1543-51.

4. Ruhl CE, Everhart JE. Gallstone disease is associated with increased mortality in the United States. Gastroenterology. 2011;140:508-16.

5. Biddinger SB, Haas JT, Yu BB, Bezy O, Jing E, Zhang W, et al. Hepatic insulin resistance directly promotes formation of cholesterol gallstones. Nat Med. 2008;14:778-82.

6. Chen YC, Chang CC, Liu KL. Mirizzi syndrome due to a large radiolucent gallstone. Am J Gastroenterol. 2016;111:599.

7. Lammert F, Miquel JF. Gallstone disease: from genes to evidence-based therapy. J Hepatol. 2008;48(Suppl 1):S124-35. 
8. van Erpecum KJ. Biliary lipids, water and cholesterol gallstones. Biol Cell. 2005;97:815-22.

9. Halpern Z, Moshkowitz M, Laufer H, Peled Y, Gilat T. Effect of phospholipids and their molecular species on cholesterol solubility and nucleation in human and model biles. Gut. 1993;34:110-5.

10. Wolfe BM, Gardiner B, Frey CF. Laparoscopic cholecystectomy: a remarkable development. JAMA. 2015;314:1406.

11. Loozen CS, Oor JE, van Ramshorst B, van Santvoort HC, Boerma D. Conservative treatment of acute cholecystitis: a systematic review and pooled analysis. Surg Endosc. 2017;31:504-15.

12. Hu M, Fok BS, Wo SK, Lee VH, Zuo Z, Tomlinson B. Effect of common polymorphisms of the farnesoid $\mathrm{x}$ receptor and bile acid transporters on the pharmacokinetics of ursodeoxycholic acid. Clin Exp Pharmacol Physiol. 2016; 43:34-40.

13. Roma MG, Toledo FD, Boaglio AC, Basiglio CL, Crocenzi FA, Sanchez PE. Ursodeoxycholic acid in cholestasis: linking action mechanisms to therapeutic applications. Clin Sci (Lond). 2011;121:523-44.

14. Chen Y, Kong J, Wu S. Cholesterol gallstone disease: focusing on the role of gallbladder. Lab Investig. 2015;95:124-31.

15. Guarino MP, Cong P, Cicala M, Alloni R, Carotti S, Behar J. Ursodeoxycholic acid improves muscle contractility and inflammation in symptomatic gallbladders with cholesterol gallstones. Gut. 2007;56:815-20.

16. Rebholz C, Krawczyk M, Lammert F. Genetics of gallstone disease. Eur J Clin Investig. 2018;48:e12935.

17. Beltowski J. Liver $x$ receptors (lxr) as therapeutic targets in dyslipidemia. Cardiovasc Ther. 2008;26:297-316.

18. Yu DD, Andrali SS, Li H, Lin M, Huang W, Forman BM. Novel fxr (farnesoid $x$ receptor) modulators: potential therapies for cholesterol gallstone disease. Bioorgan Med Chem. 2016;24:3986-93.

19. Back SS, Kim J, Choi D, Lee ES, Choi SY, Han K. Cooperative transcriptional activation of atp-binding cassette sterol transporters abcg5 and abcg8 genes by nuclear receptors including liver-x-receptor. BMB Rep. 2013;46:322-7.

20. Graf GA, Yu L, Li WP, Gerard R, Tuma PL, Cohen JC, et al. Abcg5 and abcg8 are obligate heterodimers for protein trafficking and biliary cholesterol excretion. J Biol Chem. 2003;278:48275-82.

21. Stender S, Frikke-Schmidt R, Nordestgaard BG, Tybjaerg-Hansen A. The abcg $5 / 8$ cholesterol transporter and myocardial infarction versus gallstone disease. J Am Coll Cardiol. 2014;63:2121-8.

22. Carey MC. Critical tables for calculating the cholesterol saturation of native bile. J Lipid Res. 1978;19:945.

23. Liu M, Liu C, Chen H, Huang X, Zeng X, Zhou J, et al. Prevention of cholesterol gallstone disease by schaftoside in lithogenic diet-induced c57bl/6 mouse model. Eur J Pharmacol. 2017;815:1-09.

24. Hancock-Cerutti W, Rader DJ. Opposing effects of abcg5/8 function on myocardial infarction and gallstone disease. J Am Coll Cardiol. 2014;63: 2129-30.

25. Repa JJ, Berge KE, Pomajzl C, Richardson JA, Hobbs H, Mangelsdorf DJ Regulation of atp-binding cassette sterol transporters abcg 5 and abcg 8 by the liver $x$ receptors alpha and beta. J Biol Chem. 2002;277:18793-800.

26. Yu L, Li-Hawkins J, Hammer RE, Berge KE, Horton JD, Cohen JC, et al. Overexpression of abcg5 and abcg8 promotes biliary cholesterol secretion and reduces fractional absorption of dietary cholesterol. J Clin Invest. 2002; 110:671-80.

27. Wang HH, Li X, Patel SB, Wang DQ. Evidence that the adenosine triphosphate-binding cassette g5/98-independent pathway plays a determinant role in cholesterol gallstone formation in mice. Hepatology. 2016;64:853-64

28. Song P, Rockwell CE, Cui JY, Klaassen CD. Individual bile acids have differential effects on bile acid signaling in mice. Toxicol Appl Pharm. 2015; 283:57-64.

29. Wang HH, Li X, Patel SB, Wang DQH. Evidence that the adenosine triphosphate-binding cassette g5/g8-independent pathway plays a determinant role in cholesterol gallstone formation in mice. Hepatology. 2016;64:853-64.

30. Janowski BA, Willy PJ, Devi TR, Falck JR, Mangelsdorf DJ. An oxysterol signalling pathway mediated by the nuclear receptor Ixr alpha. Nature. 1996;383:728-31.

31. Sun L, Pang Y, Wang X, Wu Q, Liu H, Liu B, et al. Ablation of gut microbiota alleviates obesity-induced hepatic steatosis and glucose intolerance by modulating bile acid metabolism in hamsters. Acta Pharm Sin B. 2019;9: 702-10.
32. Ghonem NS, Assis DN, Boyer JL. Fibrates and cholestasis. Hepatology. 2015; 62:635-43.

33. Makishima M, Okamoto AY, Repa JJ, Tu H, Learned RM, Luk A, et al. Identification of a nuclear receptor for bile acids. Science. 1999;284:1362-5.

34. Russell DW. Nuclear orphan receptors control cholesterol catabolism. Cell. 1999;97:539-42.

35. Li T, Matozel M, Boehme S, Kong B, Nilsson LM, Guo G, et al. Overexpression of cholesterol 7alpha-hydroxylase promotes hepatic bile acid synthesis and secretion and maintains cholesterol homeostasis. Hepatology. 2011;53:9961006.

36. Marrapodi M, Chiang JY. Peroxisome proliferator-activated receptor alpha (pparalpha) and agonist inhibit cholesterol 7alpha-hydroxylase gene (cyp7a1) transcription. J Lipid Res. 2000;41:514-20.

37. Post SM, Duez H, Gervois PP, Staels B, Kuipers F, Princen HM. Fibrates suppress bile acid synthesis via peroxisome proliferator-activated receptoralpha-mediated downregulation of cholesterol 7alpha-hydroxylase and sterol 27-hydroxylase expression. Arterioscler Thromb Vasc Biol. 2001;21: 1840-5.

38. Xie Y, Newberry EP, Kennedy SM, Luo J, Davidson NO. Increased susceptibility to diet-induced gallstones in liver fatty acid binding protein knockout mice. J Lipid Res. 2009;50:977-87.

39. Houten SM, Volle DH, Cummins CL, Mangelsdorf DJ, Auwerx J. In vivo imaging of farnesoid $x$ receptor activity reveals the ileum as the primary bile acid signaling tissue. Mol Endocrinol. 2007:21:1312-23.

40. Houten SM, Watanabe M, Auwerx J. Endocrine functions of bile acids. EMBO J. 2006;25:1419-25.

41. Fukumoto $\mathrm{S}$. Actions and mode of actions of fgf19 subfamily members. Endocr J. 2008:55:23-31.

42. Zhang Y, Jiang R, Zheng X, Lei S, Huang F, Xie G, et al. Ursodeoxycholic acid accelerates bile acid enterohepatic circulation. Brit J Pharmacol. 2019;176: 2848-63.

43. Mueller M, Thorell A, Claudel T, Jha P, Koefeler H, Lackner C, et al. Ursodeoxycholic acid exerts farnesoid $\mathrm{x}$ receptor-antagonistic effects on bile acid and lipid metabolism in morbid obesity. J Hepatol. 2015;62:1398-404.

44. Sun L, Xie C, Wang G, Wu Y, Wu Q, Wang X, et al. Gut microbiota and intestinal fxr mediate the clinical benefits of metformin. Nat Med. 2018;24: 1919-29.

45. Kubitz R, Dröge C, Stindt J, Weissenberger K, Häussinger D. The bile salt export pump (bsep) in health and disease. Clin Res Hepatol Gas. 2012;36: $536-53$.

46. Mataki C, Magnier BC, Houten SM, Annicotte J, Argmann C, Thomas C, et al. Compromised intestinal lipid absorption in mice with a liver-specific deficiency of liver receptor homolog 1. Mol Cell Biol. 2007;27:8330-9.

47. Weerachayaphorn J, Cai S, Soroka CJ, Boyer JL. Nf-e2- related factor 2 (nrf2) is a positive regulator of human bile salt export pump (bsep) expression. Hepatology. 2009;50:1588-96.

48. Wang HH, Lammert F, Schmitz A, Wang DQ. Transgenic overexpression of abcb11 enhances biliary bile salt outputs, but does not affect cholesterol cholelithogenesis in mice. Eur J Clin Investig. 2010;40:541-51.

49. Henkel AS, Karin ERG, Havinga R, Boverhof R, Green RM, Verkade HJ. Hepatic overexpression of abcb11 in mice promotes the conservation of bile acids within the enterohepatic circulation. Am J Physiol Gastrointest Liver Physiol. 2013;304:221-6.

50. Li T, Chiang JY. Bile acid signaling in metabolic disease and drug therapy. Pharmacol Rev. 2014;66:948-83.

51. Krawczyk M, Wang DQ, Portincasa P, Lammert F. Dissecting the genetic heterogeneity of gallbladder stone formation. Semin Liver Dis. 2011;31:15772.

52. Lammert F, Carey MC, Paigen B. Chromosomal organization of candidate genes involved in cholesterol gallstone formation: a murine gallstone map. Gastroenterology. 2001;120:221-38.

\section{Publisher's Note}

Springer Nature remains neutral with regard to jurisdictional claims in published maps and institutional affiliations. 